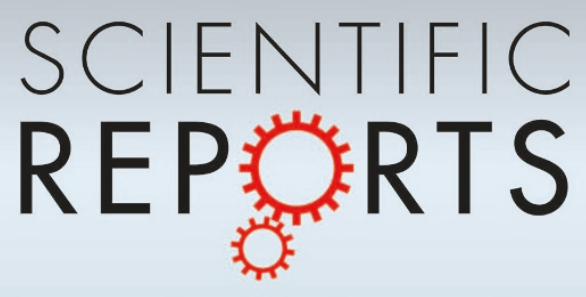

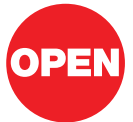

SUBJECT AREAS:

MOLECULAR SELF-

ASSEMBLY

STRUCTURAL PROPERTIES

SELF-ASSEMBLY

GELS AND HYDROGELS

Received

5 April 2013

Accepted

29 April 2013

Published

13 May 2013

Correspondence and requests for materials should be addressed to X.W. (wangxun@mail. tsinghua.edu.cn)

\section{Polyoxometalate-based Supramolecular Gel}

\author{
Peilei He, Biao Xu, Huiling Liu, Su He, Faisal Saleem \& Xun Wang \\ Department of Chemistry, Tsinghua University, Beijing 100084, P. R. China.
}

Self-assemblyings of surfactant-encapsulated Wells-Dawson polyoxometalates (SEPs) nanobuilding blocks in butanone and esters yielded supramolecular gels showing thermo and photo responsive properties. The gels can be further polymerized if unsaturated esters were used and subsequently electrospinned into nanowires and non-woven mats. The as-prepared non-woven mats have a Young's modulus as high as $542.55 \mathrm{MPa}$. It is believed that this supramolecular gel is a good platform for polyoxometalates processing.

olyoxometalates (POMs) are discrete molecular oxides of early transition metals ${ }^{1}$ and have obtained widespread application ${ }^{2-6}$ due to their advantageous properties, e.g., photochromism ${ }^{4,7}$, electrochromism ${ }^{4,8}$, magnetism $^{9}$, catalysis ${ }^{5,10}$, chirality ${ }^{11}$. Till now, most of the studies have been focused on the growth of macrosized single crystals of POMs ${ }^{12-15}$ as their structures can be solved ${ }^{16}$. For all the success in the designs and findings of new type of POMs, these are still inherently large anions that are hard to process directly ${ }^{17}$. Great efforts have been made to build interfaces between POM anions and their surroundings to make them practically useful ${ }^{18-22}$. Most of the research centres are using covalent modification ${ }^{23-27}$ and non-covalent modification ${ }^{28-30}$ to build bridge between the POM and organic molecules. Compared with the covalent approach, non-covalent modification is easier to purify final system and get higher productivity.

Non-covalent surface modification uses the cation of quaternary ammonium salt to encapsulate POMs and transfer them from aqueous to nonaqueous phase. In the last few years, self-assemblyings of Keggin-type ${ }^{29,30}$ and Anderson-type ${ }^{31,32}$ POM clusters based on non-covalent modification have been explored. In addition, this modification strategy provides the possibilities for fast and controlled self-assembly of POMs into nanostructures $^{33}$, which maybe have a great potential to become candidate materials. So far, many nanostructures of surfactants encapsulate polyoxometalates assemblies, such as microspheres ${ }^{29}$, nanofibers ${ }^{34}$, nanodisks $s^{30}$, nanoflower ${ }^{35}$, nanocones and nanotubes ${ }^{30}$, have been achieved. Recently, we succeeded in the fabrication of nanocone that showed high-efficiency and selectivity toward the catalytic oxidation of sulphides when used as a nanoreactor $^{36}$. Other than that, most of the assemblies failed to become available materials. A lot of efforts will be needed in the processing and applications of POMs materials.

Herein, we report the formation and response of a supramolecular gel by the self-assembly of surfactant encapsulated Wells-Dawson polyoxometalates which occurred in the butanone and ethyl acetate. In addition, ethyl acetate could be substituted by other unsaturated esters with carbon-carbon double bond or esters with other functional groups such as photofunctional groups. In this paper, we have tried the unsaturated ester. When the ester was substituted by unsaturated ester, the gel could be polymerized. In fact, it builds interface between the Wells-Dawson POM and the polymer. Photochromic hybrid polymer was obtained when the unsaturated ester was polymerized completely. Non-woven mats with greater mechanical stress were fabricated by electrospinning the partially polymerized gel. Therefore, we believe that supramolecular gel is a good platform for polyoxometalates processing.

\section{Results}

Firstly, the SEPs were synthesized by using the reported two-phase method ${ }^{29}$. The biggest difference was the use of two surfactants. Hexadecyl trimethyl ammonium bromide (CTAB) and tetrabutyl ammonium bromide (TBAB) were used to encapsulate the polyoxometalates with characteristic crystal structure of Wells-Dawson $\left(\mathrm{K}_{6} \mathrm{P}_{2} \mathrm{~W}_{18} \mathrm{O}_{62} \cdot \mathrm{nH}_{2} \mathrm{O}\right)$. In order to determine the component of SEPs, we took the SEPs for thermal gravimetric analysis (TGA) and NMR characterization. Figure 1a shows that the weight loss of SEPs is about $28.17 \%$ from 20 to $900^{\circ} \mathrm{C}$. To combine the reference ${ }^{29}$ and TGA results, we think that the six potassium ions have been totally substituted by the cation of quaternary ammonium salt. There are six surfactants molecules in the SEPs. From the ${ }^{1} \mathrm{H}$ NMR spectrum (Figure 1b, the whole ${ }^{1} \mathrm{H}$ NMR spectra was shown in Figure S1), the integral area of $\mathrm{H}-\mathrm{a}$ 

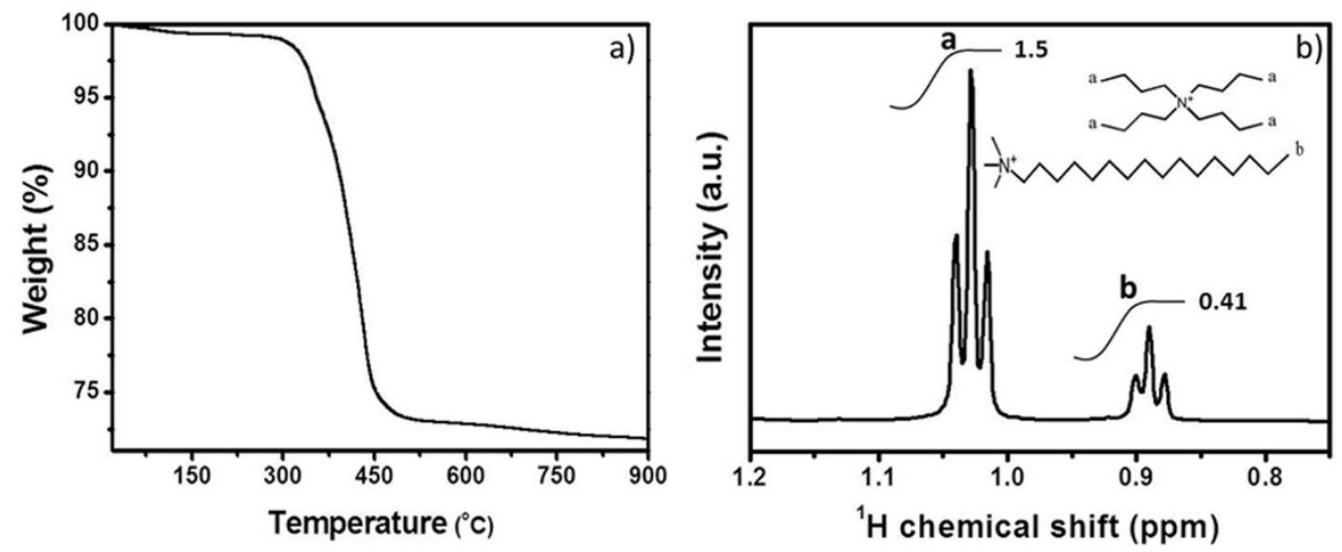

Figure $1 \mid$ (a) TGA of SEPs in the nitrogen atmosphere with a scanning rate of $10^{\circ} \mathrm{C} \mathrm{min}^{-1}$. (b) ${ }^{1} \mathrm{H}$ NMR spectra of the pure $\mathrm{SEPs}$ in $\mathrm{C}_{3} \mathrm{D}_{6} \mathrm{O}$.

hydrogen $(1.03 \mathrm{ppm})$ in TBAB is 1.5 and $\mathrm{H}$-b hydrogen $(0.89 \mathrm{ppm})$ in CTAB is 0.41 . There are four $\mathrm{H}$-a hydrogen in one TBAB molecule. We can calculate the ratio of CTAB and TBAB is $0.9: 1$ which is close to $1: 1$. So, we can conclude the chemical formula of SEPs is

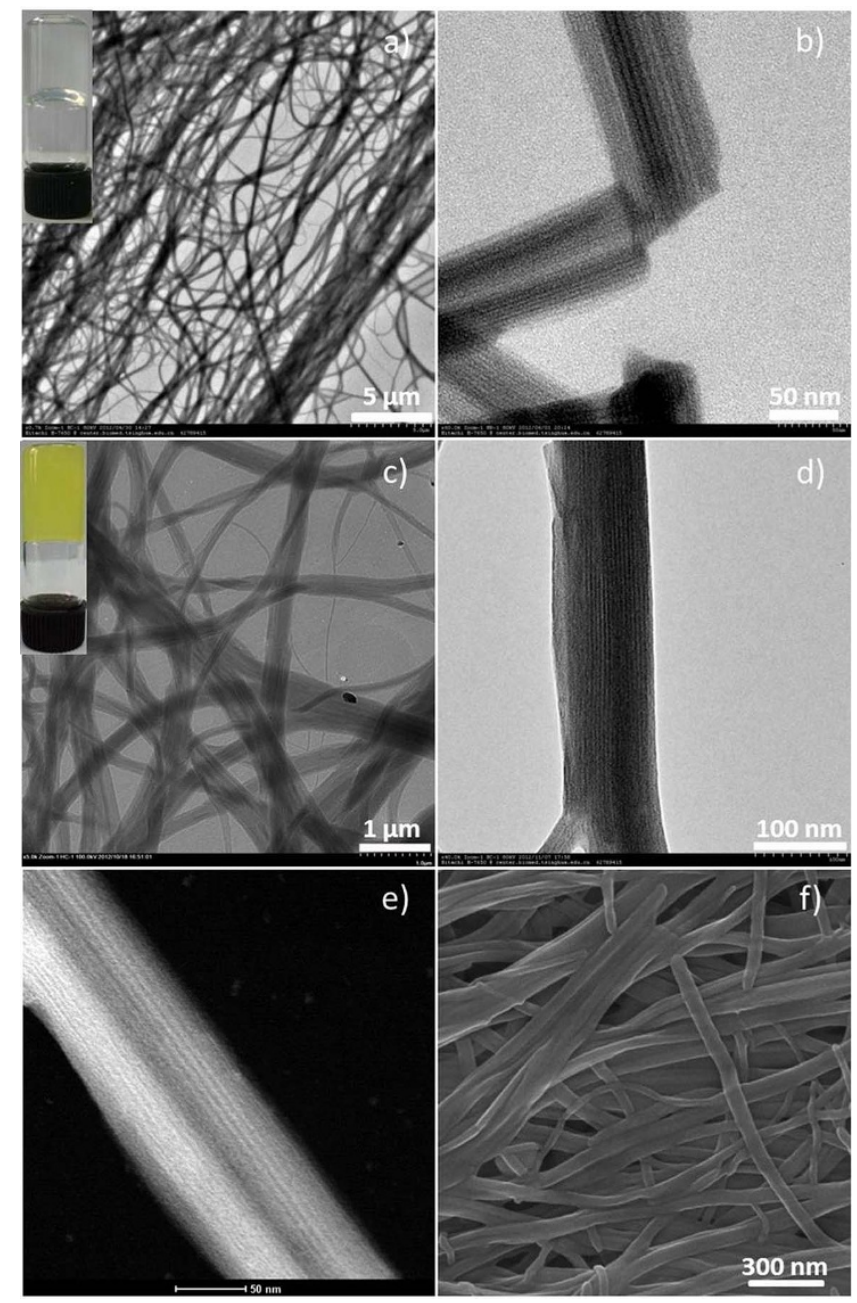

Figure $2 \mid(\mathrm{a}-\mathrm{b})$ TEM images of nanowires which were made up of $(\mathrm{CTA})_{3}(\mathrm{TBA})_{3} \mathrm{P}_{2} \mathrm{~W}_{18} \mathrm{O}_{62}$. The inset shows the photo of gel formed by nanowires. (c-d) TEM images of nanowires which were made up of $(\mathrm{CTA})_{3}(\mathrm{TBA})_{3} \mathrm{P}_{2} \mathrm{Mo}_{18} \mathrm{O}_{62}$. The inset shows the photo of gel. (e) (highangle annular dark-field scanning TEM) HAADF-STEM image of nanowires. (f) SEM image of nanowires.
$(\mathrm{CTA})_{3}(\mathrm{TBA})_{3} \mathrm{P}_{2} \mathrm{~W}_{18} \mathrm{O}_{62}$. In addition, Figure $\mathrm{S} 2$ shows the TEM images of SEPs which are dispersed in butanone. The diameter of SEPs is about $1 \sim 2 \mathrm{~nm}$ which is the limitation of resolution in TEM. Therefore, it is difficult to pick a particle of SEPs out.

After that we studied the self-assembly behaviour of SEPs. The butanone solution of SEPs changed from transparent to opaque with ester added to it. When the ratio of butanone to ethyl acetate is $2: 1$, we can get a transparent gel. Originally, the solution was opaque when the ethyl acetate was added. But, the opaque solution became a transparent gel in three minutes. We can see that the formation of gel is very fast. TEM and SEM images (Figure $2 \mathrm{a}, \mathrm{b}, \mathrm{c}, \mathrm{d}, \mathrm{f}$ ) show the gel consists of nanowires. The length of nanowires is nearly dozens of micrometres. Furthermore, the nanowires have a lamellar structure which can be seen from the magnified TEM image (Figure $2 \mathrm{~b}$ ). The same result can be obtained from HADDF-STEM images (Figure 2c). By measuring the interlayer space in TEM image (Figure $2 b$ ), we found that the interlayer space is about $3 \mathrm{~nm}$. X-ray energy dispersive spectroscopy (EDS) with HRTEM indicates the existence of nitrogen and tungsten all over the nanowires, which shows that the nanowires are composed of SEPs (Figure S3).

To verify the structure of the nanowires, we slice them to observe the cross section of the nanowires under TEM characterization. We selected epoxy resin as embedding medium. Then curing process proceeded at $60^{\circ} \mathrm{C}$. The slice process carried out under room temperature and cryoprocess wasn't needed. Figure 3a shows that the nanowires are circular. X-ray diffraction pattern (Figure 3c) of dried assemblies showed a layer spacing $3.27 \mathrm{~nm}$ which is in accordance with the result of TEM (Figure 2b). When the ethyl acetate was replaced by other esters, the sol could also be transformed into gel. And the structure of nanowires could be maintained. The photo of some typical gels is shown in Figure S4. When the esters were unsaturated esters having carbon-carbon double bond or esters with other functional groups, the gel would have more functionality. Thus this supramolecular gel is a good platform for POMs processing.

The thermo and photo responsive behavior of the gel have been also studied. As demonstrated in Figure 4, we found that the gel transformed into sol when the gel was shaken, suggesting that the gel has a poor stability. Then we heated the sol to $70^{\circ} \mathrm{C}$, which is close to the boiling point of the solvents, for one hour. The sol was again transformed into gel. Therefore, the hybrid gel was thermally stable. This behavior of the gel is similar to that of the copolymer gel $^{37}$. Figure S5 shows the photochromic process of the gel. When the gel was irradiated for two hours, some blue dots occurred in the gel. With an increase in the irradiation time, the gel became dark blue and part of the gel shrunk to sol. The color of gel faded when it stayed for three hours in the air. The photochromic process is caused by the photo reduction of tungsten ${ }^{7}$. 

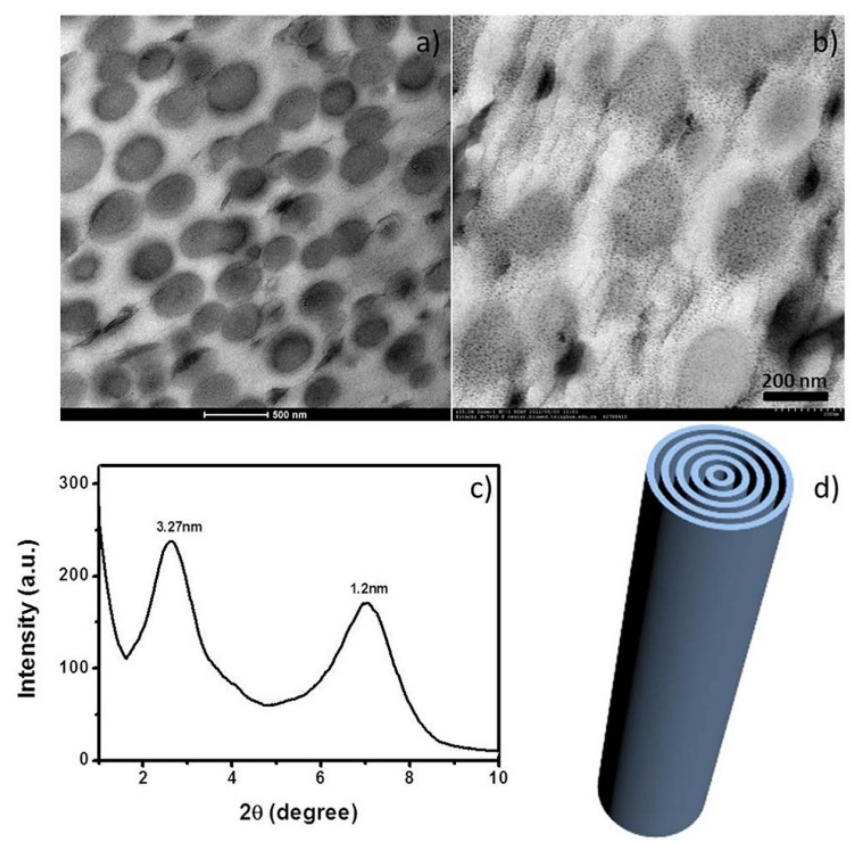

Figure 3 (a) TEM image of the cross section of the nanowires. (b) Magnified TEM images of the cross section. (c) Small Angle X-ray Diffraction of the nanowires. (d) Schematic drawing of the nanoroll.

Then we used methyl methacrylate instead of ethyl acetate to form the gel, and a little amount of polymerization initiator was added into the methyl methacrylate. Then the polymerization of the gel occurred in the oven at $70^{\circ} \mathrm{C}$. Afterwards we got the hybrid polymer (the inset photo of Figure S6) with POMs. Figure S6 shows the cross section of the hybrid polymer. The bright dots should be the cross section of the nanowires (Figure S6) and the hybrid polymer could keep the good reversible photochromism. Figure 5 shows the photochromic property of the hybrid polymer. As shown in the Figure 5a, when the hybrid polymer was irradiated for three hours, it became dark blue. The transmittance spectrum of the hybrid polymer before and after irradiation is shown in Figure $5 \mathrm{c}$. When the blue polymer was stored in dark, the color of the polymer faded after 24 hours. It is obvious that the decoloration of gel is much faster than the polymer and the color change is reversible (Figure $5 c$ ). When the blue polymer was put into nitrogen atmosphere, the decoloration was much slower. On the basis of above results, we believe that oxygen is the

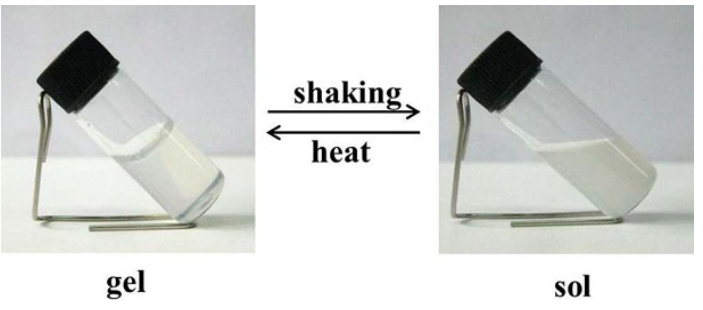

Figure $4 \mid$ Photo of the thermo responsive behavior of the gel.

key factor in decoloration. The much more oxygen in the gel than in the polymer leads to the much faster decoloration of the gel $^{7}$.

Since the gel can be polymerized when unsaturated ester replaced ethyl acetate, we tried to use electrospinning to process the gel. Although the immediately-formed gel has a low viscosity, it cannot be used for electrospinning directly. But we can still get a sticky hybrid polymer gel by polymerizing the gel.

According to the responsive behavior of gel, it can be transformed into sol when it is shaken. Then we got a spinnable feeding solution. After electrospinning, we got non-woven mats (optical images in Figure 6a,b) which consisted of ribbon-like fibers (Figure 6a,b). The possible reason for the formation of ribbon-like structure (Figure 6c) is that the concentration of polymer is too high ${ }^{38}$. Because of our good platform, it is much easier to get other morphologies of fiber through adjusting the time of polymerization. To characterize the mechanical properties of the non-woven mats, we used a wheel-shaped collector to get parallel fibers (Figure 6d) at $1600 \mathrm{rpm}^{39}$. We studied the stress-strain behavior of the parallel fibers. In the tests, the orientation of the applied stress is parallel to the fiber arrangement direction. The loading speed is $1 \mathrm{~mm} / \mathrm{min}$. The tests were carried out at the room temperature which is about $20^{\circ} \mathrm{C}$. The stress-strain curves of the fibrous membranes are shown in Figure 6. Young's modulus of the two membranes is $427.18 \mathrm{MPa}$ (Figure 6e) and 542.55 MPa (Figure 6f). The Young's modulus values are all the average values within the elastic range. From these results it seems very clear that the good platform of supramolecular gel provides many advantages in the electrospinning.

\section{Discussion}

In order to find out the gel formation process, we carried a time control experiment. During the formation of gel, we took the samples for TEM characterization at different times. Figure S7a was the TEM image when ethyl acetate had been added into butanone for $20 \mathrm{~s}$.

a)
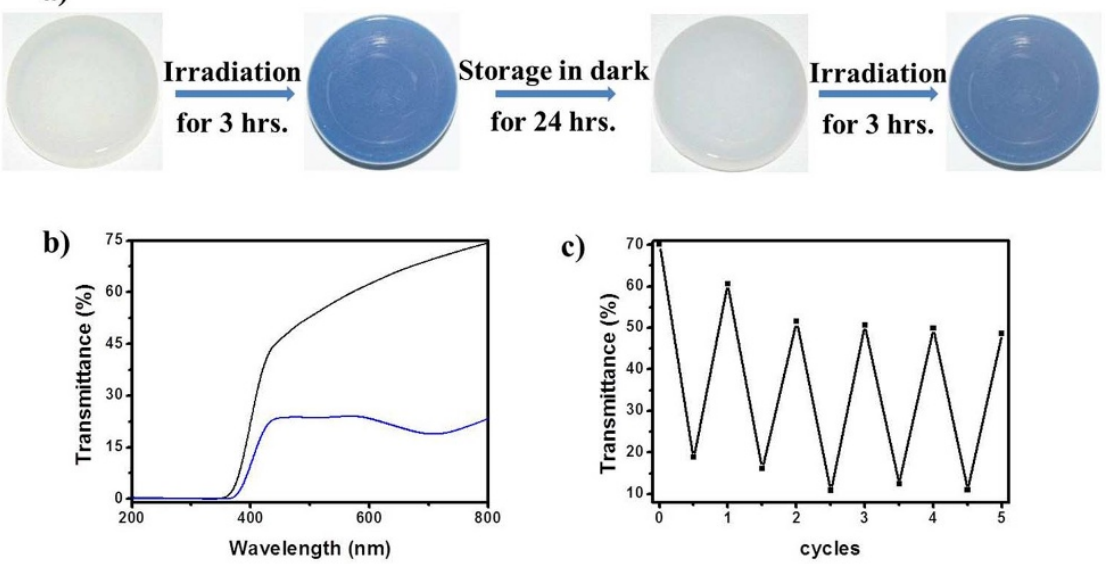

c)

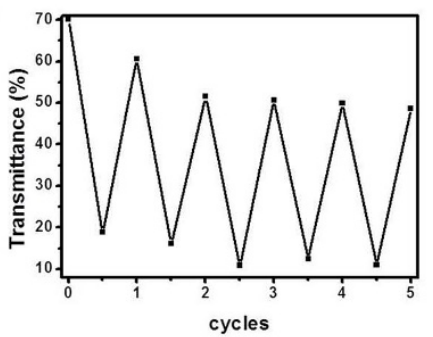

Figure $5 \mid$ (a) Reversible photochromism property of the hybrid polymer when irradiated with UV light (The hybrid polymer got from the polymerization of gel). (b) Transmittance spectra of the hybrid polymer before (black line) and after (blue line) irradiation. (c) The photochromic switching of the transmittance change (monitored at $715 \mathrm{~nm}$ ) during consecutive cycles of UV irradiation and storage in the dark. 

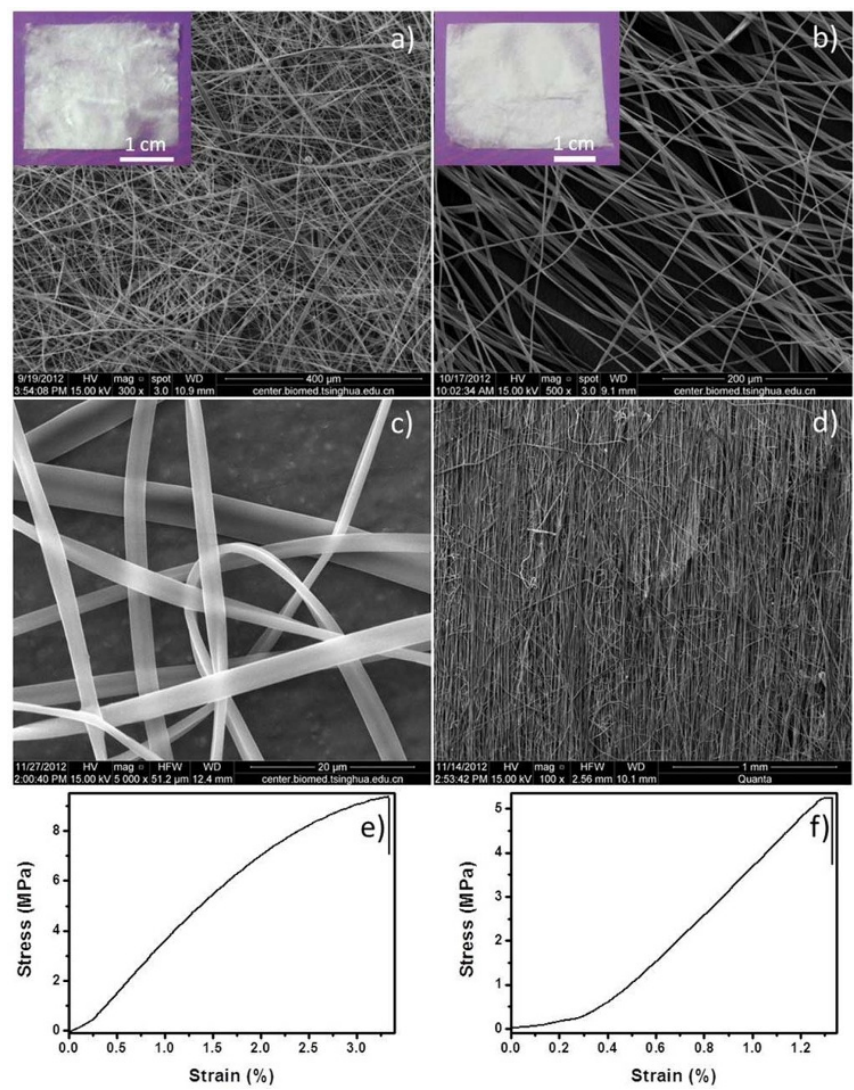

Figure 6 | SEM images and photographs of the fiber mats with different polyoxometalates gel. (a) $\mathrm{CTA}_{3} \mathrm{TBA}_{3} \mathrm{P}_{2} \mathrm{~W}_{18} \mathrm{O}_{62}$ (b) $\mathrm{CTA}_{3} \mathrm{TBA}_{3}$ $\mathrm{P}_{2} \mathrm{Mo}_{18} \mathrm{O}_{62}$. (c) Magnified SEM images of the fibers. (d) SEM image of parallel fibers. Stress-strain curves of fibrous membrane prepared by polymethyl methacrylate with (e) $\mathrm{CTA}_{3} \mathrm{TBA}_{3} \mathrm{P}_{2} \mathrm{~W}_{18} \mathrm{O}_{62}$ (f) $\mathrm{CTA}_{3} \mathrm{TBA}_{3}$ $\mathrm{P}_{2} \mathrm{Mo}_{18} \mathrm{O}_{62}$.

Many spheres and a few nanowires occurred. Ethyl acetate as a poor solvent resulted in the aggregation of SEPs. And this can explain why many spheres occurred in the opaque solution. After $40 \mathrm{~s}$, the amount of nanowires increased and the amount of spheres decreased slowly (as shown in Figure S7b) and the flowability of the solution decreased. When ethyl acetate had been added for $60 \mathrm{~s}$, more and more nanowires occurred. When the time was increased to $2 \mathrm{~min}$, we could see the sample was constituted of nanowires and spheres in the TEM image (Figure S7d). After $3 \mathrm{~min}$, the transparent gel (the inset photo of Figure 2a) formed and the TEM image was the same as Figure 2a. These results indicate the aggregations or assemblies in the solution change from spheres to nanowires. It is corresponding to the change from opaque solution to transparent gel. This process was caused by the rearrangement or self-assembly of SEPs. The diameter of nanowires is less than $100 \mathrm{~nm}$, so the gel is transparent.

Moreover, other polyoxometalates have been tried to form gel. First of all, Wells-Dawson phosphomolybic acid $\left(\mathrm{H}_{6} \mathrm{P}_{2} \mathrm{Mo}_{18} \mathrm{O}_{62}\right)$ was used to do the self-assembly. In the same condition, we got a yellow gel (the inset of Figure 2c) and TEM images (Figure 2c, d) show that the gel consists of nanorolls. But all attempts to Keggin POMs ended in failure. The results revealed that the structure of Wells-Dawson POM played a very important role in forming gel. We also tried to use monolacunary Wells-Dawson $\left(\mathrm{K}_{10} \mathrm{P}_{2} \mathrm{~W}_{17} \mathrm{O}_{61}\right.$. $\left.\mathrm{nH}_{2} \mathrm{O}\right)^{40}$ and monosubstituted Wells-Dawson $\left(\mathrm{K}_{7} \mathrm{P}_{2} \mathrm{~W}_{17} \mathrm{VO}_{62}{ }^{\circ}\right.$ $\left.\mathrm{nH}_{2} \mathrm{O}\right)^{41}$ to form gel. Unfortunately, no gel was formed. So in the formation of gel, ideal Wells-Dawson POMs is essential. An alternative explanation is that the surface charge of monolacunary and monosubstituted Wells-Dawson POMs changed. We believe that all ideal Wells-Dawson POMs can be used to form gel besides phosphotungstate and phosphomolybic acid.

According to the XRD result, we can conclude the nanowires have a lamellar structure. To combine with the lamellar structure and the circular shape (Figure 3a, b), we think that nanorolls are more exact to describe the structure of assemblies than nanowires. And Figure $3 \mathrm{~d}$ is a brief scheme of nanoroll.

We have proposed a hypothesis to explain why the SEPs can form gel by self-assembly. First, the SEPs self-assemble into long nanorolls. Then the nanorolls' entanglement subsequently leads to gel formation. This hypothesis could be supported by the time control experiments. As shown in Figure 7, the three-dimensional network in the gel was made up of nanorolls. The nanorolls were obtained from the self-assembly of the SEPs. As we mentioned above, one Wells-Dawson POM cluster was encapsulated by three CTAB molecules and three TBAB molecules. CTAB and TBAB molecules spaced at regular intervals. This kind of distribution leads to the SEPs rolls

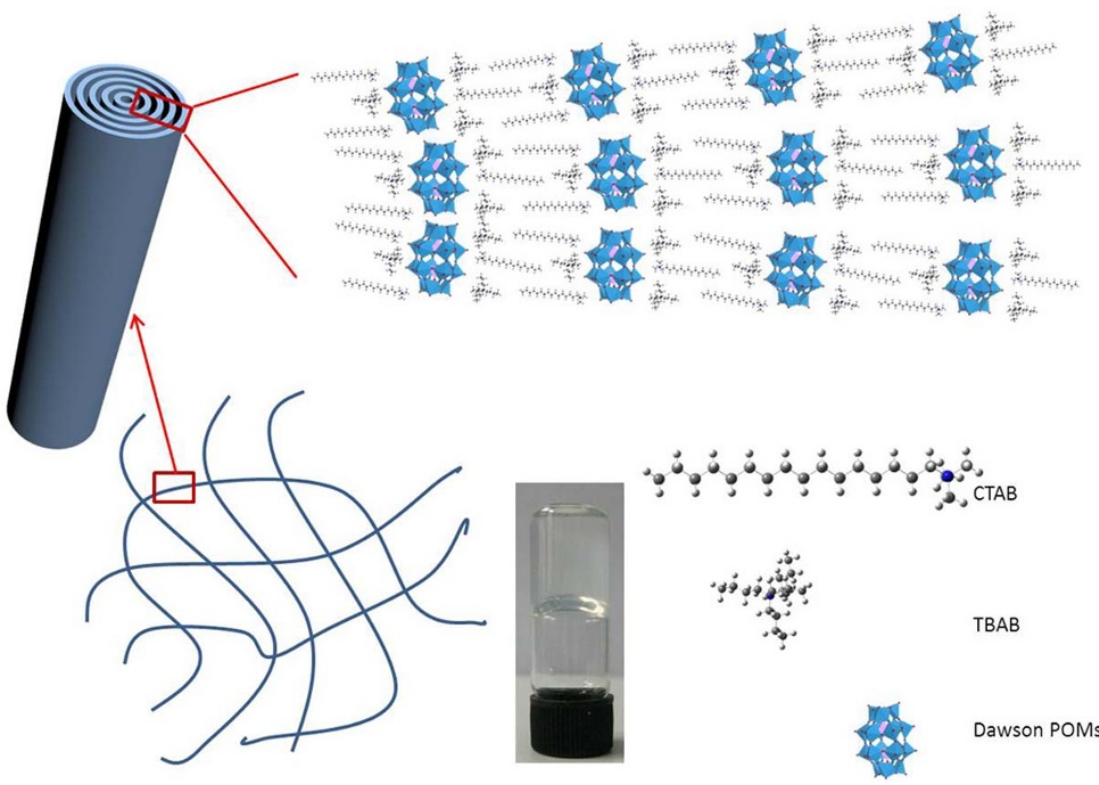

Figure $7 \mid$ A schematic representation of the possible formation of the transparent gel. 
up to form nanorolls. The interaction between nanorolls may be the van der Waals force. However, this is difficult to test and verify.

In summary, by self-assembly we got a hybrid gel which was made up of nanorolls with lamellar structure. The gel was formed in 3 minutes, and it had thermo-responsive and photochromic properties as well. At the same time, the esters in this system could be substituted by unsaturated esters. So, the gel was very easy to be polymerized. Based on the fast formation and polymerizable properties of the gel, we got hybrid polymer. At the same time, the photochromic property and structure could also be preserved. Non-woven mats with greater mechanical stress were fabricated by electrospinning of the partially polymerized gel. Therefore, we believe that this supramolecular gel is a good platform for polyoxometalates processing.

\section{Methods}

Synthesis of SEPs. CTAB $(0.91 \mathrm{~g}, 2.5 \mathrm{mmol})$ and TBAB $(0.81 \mathrm{~g}, 2.5 \mathrm{mmol})$ were added into $\mathrm{CHCl}_{3}(20 \mathrm{~mL})$, either $\mathrm{K}_{6} \mathrm{P}_{2} \mathrm{~W}_{18} \mathrm{O}_{62} \cdot \mathrm{nH}_{2} \mathrm{O}$ or $\mathrm{H}_{6} \mathrm{P}_{2} \mathrm{~W}_{18} \mathrm{O}_{62} \cdot \mathrm{nH}_{2} \mathrm{O}$ $\left(0.8 \mathrm{mmol}\right.$, synthesized according to reference $\left.{ }^{42,43}\right)$ was added into water $(10 \mathrm{~mL})$ After that, the organic phase was added into the solution of polyoxometalates with stirring. After one hour, the mixture was centrifuged at $10000 \mathrm{rpm}$ for $8 \mathrm{~min}$. Then the precipitates were washed 2 times by deionized water. In the end, the precipitates were dried at room temperature. The product was obtained as some powder.

Self-assembly. The product $(20 \mathrm{mg})$ was dispersed in butanone $(2 \mathrm{~mL})$. Then ethyl acetate or other esters $(1 \mathrm{~mL})$ was added into the solution of butanone at room temperature. The ratio of butanone and esters changed when we used different esters in self-assembly.

Polymerization. First, benzoyl peroxide (BPO, $20 \mathrm{mg}$ ) was added into methyl methacrylate $(1 \mathrm{~mL})$. It was added into the butanone solution $(1 \mathrm{~mL})$ of SEPs. Methyl methacrylate was substituted for ethyl acetate to do the self-assembly. Then the assemblies were kept in the oven at $70^{\circ} \mathrm{C}$ for several hours.

Electrospinning. The gel with partial polymerization was shaken to become sol and it was loaded into a plastic syringe with a stainless steel needle. A piece of flat aluminium foil was used as an acceptor. The distance between the needle and the acceptor was $10 \mathrm{~cm}$. And the voltage was $13 \mathrm{kV}$. The flow rate was about $1 \mathrm{~mL} / \mathrm{h}$. The electrospinning process was conducted in air. A wheel-shaped collector was used to get parallel fibers at $1600 \mathrm{rpm}$. The voltage and distance didn't change.

1. Cooper, G. J. T. et al. Osmotically driven crystal morphogenesis: A general approach to the fabrication of micrometer-scale tubular architectures based on polyoxometalates. J. Am. Chem. Soc 133, 5947-5954 (2011).

2. Zeng, H., Newkome, G. R. \& Hill, C. L. Poly(polyoxometalate) dendrimers: Molecular prototypes of new catalytic materials. Angew. Chem. Int. Ed. 39, 1771-1774 (2000).

3. Katsoulis, D. E. A survey of applications of polyoxometalates. Chem. Rev. $\mathbf{9 8}$ 359-388 (1998).

4. Yamase, T. Photo- and electrochromism of polyoxometalates and related materials. Chem. Rev. 98, 307-326 (1998).

5. Kozhevnikov, I. V. Catalysis by heteropoly acids and multicomponent polyoxometalates in liquid-phase reactions. Chem. Rev. 98, 171-198 (1998).

6. Fan, D., Jia, X., Tang, P., Hao, J. \& Liu, T. Self-patterning of hydrophobic materials into highly ordered honeycomb nanostructures at the air/water interface. Angew. Chem. Int. Ed. 46, 3342-3345 (2007).

7. He, T. \& Yao, J. Photochromism in composite and hybrid materials based on transition-metal oxides and polyoxometalates. Prog. Mater. Sci. 51, 810-879 (2006).

8. Zhang, T., Liu, S., Kurth, D. G. \& Faul, C. F. J. Organized nanostructured complexes of polyoxometalates and surfactants that exhibit photoluminescence and electrochromism. Adv. Funct. Mater. 19, 642-652 (2009).

9. Clemente-Juan, J. M., Coronado, E., Galán-Mascarós, J. R. \& Gómez-García, C. J. Increasing the nuclearity of magnetic polyoxometalates. Syntheses, structures, and magnetic properties of salts of the heteropoly complexes $\left[\mathrm{Ni}_{3}\left(\mathrm{H}_{2} \mathrm{O}\right)_{3}\left(\mathrm{PW}_{10} \mathrm{O}_{39}\right) \mathrm{H}_{2} \mathrm{O}\right]^{7-},\left[\mathrm{Ni}_{4}\left(\mathrm{H}_{2} \mathrm{O}\right)_{2}\left(\mathrm{PW}_{9} \mathrm{O}_{34}\right)_{2}\right]^{10-}$, and $\left[\mathrm{Ni}_{9}(\mathrm{OH})_{3}(\mathrm{H} 2 \mathrm{O})_{6}\left(\mathrm{HPO}_{4}\right)_{2}\left(\mathrm{PW}_{9} \mathrm{O}_{34}\right)_{3}\right]^{16-}$. Inorg. Chem. 38, 55-63 (1998).

10. Yin, Q. et al. A fast soluble carbon-free molecular water oxidation catalyst based on abundant metals. Science 328, 342-345 (2010).

11. Hasenknopf, B. et al. Chirality in polyoxometalate chemistry. Eur. J. Inorg. Chem. 2008, 5001-5013 (2008).

12. Long, D.-L., Burkholder, E. \& Cronin, L. Polyoxometalate clusters, nanostructures and materials: From self assembly to designer materials and devices. Chem. Soc. Rev. 36, 105-121 (2007).

13. Du, D.-Y. et al. Polyoxometalate-based crystalline tubular microreactor: Redoxactive inorganic-organic hybrid materials producing gold nanoparticles and catalytic properties. Chem. Sci. 3, 705-710 (2012).
14. Long, D.-L., Tsunashima, R. \& Cronin, L. Polyoxometalates: Building blocks for functional nanoscale systems. Angew. Chem. Int. Ed. 49, 1736-1758 (2010).

15. Sadakane, M., Dickman, M. H. \& Pope, M. T. Controlled assembly of polyoxometalate chains from lacunary building blocks and lanthanide-cation linkers. Angew. Chem. Int. Ed. 39, 2914-2916 (2000).

16. Song, Y.-F. \& Tsunashima, R. Recent advances on polyoxometalate-based molecular and composite materials. Chem. Soc. Rev. 41, 7384-7402 (2012).

17. Proust, A. et al. Functionalization and post-functionalization: A step towards polyoxometalate-based materials. Chem. Soc. Rev. 41, 7605-7622 (2012).

18. Song, J. et al. A multiunit catalyst with synergistic stability and reactivity: A polyoxometalate-metal organic framework for aerobic decontamination. J. Am. Chem. Soc. 133, 16839-16846 (2011).

19. Tian, A.-x. et al. Assembly of the highest connectivity Wells-Dawson polyoxometalate coordination polymer: The use of organic ligand flexibility. Inorg. Chem. 47, 3274-3283 (2008).

20. Clemente-León, M., Coronado, E., Delhaes, P., Gómez-García, C. J. \& Mingotaud, C. Hybrid langmuir-blodgett films formed by alternating layers of magnetic polyoxometalate clusters and organic donor molecules-towards the preparation of multifunctional molecular materials. Adv. Mater. 13, 574-577 (2001).

21. Bu, W., Li, H., Sun, H., Yin, S. \& Wu, L. Polyoxometalate-based vesicle and its honeycomb architectures on solid surfaces. J. Am. Chem. Soc. 127, 8016-8017 (2005).

22. Yin, P., Li, D. \& Liu, T. Solution behaviors and self-assembly of polyoxometalates as models of macroions and amphiphilic polyoxometalate-organic hybrids as novel surfactants. Chem. Soc. Rev. 41, 7368-7383 (2012)

23. Wei, Y., Xu, B., Barnes, C. L. \& Peng, Z. An efficient and convenient reaction protocol to organoimido derivatives of polyoxometalates. J. Am. Chem. Soc. 123, 4083-4084 (2001).

24. Bareyt, S. et al. Highly efficient peptide bond formation to functionalized wells-dawson-type polyoxotungstates. Angew. Chem. Int. Ed. 42, 3404-3406 (2003).

25. Dolbecq, A., Dumas, E., Mayer, C. d. R. \& Mialane, P. Hybrid organic-inorganic polyoxometalate compounds: From structural diversity to applications. Chem. Rev. 110, 6009-6048 (2010).

26. Yin, P. et al. Controllable self-assembly of organic-inorganic amphiphiles containing dawson polyoxometalate clusters. Chem. Eur. J. 18, 8157-8162 (2012).

27. Zhang, J., Song, Y.-F., Cronin, L. \& Liu, T. Self-assembly of organic-inorganic hybrid amphiphilic surfactants with large polyoxometalates as polar head groups. J. Am. Chem. Soc 130, 14408-14409 (2008)

28. Volkmer, D. et al. Toward nanodevices: Synthesis and characterization of the nanoporous surfactant-encapsulated keplerate (DODA $)_{40}\left(\mathrm{NH}_{4}\right)_{2}\left[\left(\mathrm{H}_{2} \mathrm{O}\right) \mathrm{nC}\right.$ $\left.\mathrm{Mo}_{132} \mathrm{O}_{372}\left(\mathrm{CH}_{3} \mathrm{COO}\right)_{30}\left(\mathrm{H}_{2} \mathrm{O}\right)_{72}\right]$. J. Am. Chem. Soc. 122, 1995-1998 (2000).

29. Li, H., Sun, H., Qi, W., Xu, M. \& Wu, L. Onionlike hybrid assemblies based on surfactant-encapsulated polyoxometalates. Angew. Chem. Int. Ed. 46, 1300-1303 (2007).

30. Nisar, A., Zhuang, J. \& Wang, X. Cluster-based self-assembly: Reversible formation of polyoxometalate nanocones and nanotubes. Chem. Mater. 21 3745-3751 (2009).

31. Yan, Y. et al. Smart self-assemblies based on a surfactant-encapsulated photoresponsive polyoxometalate complex. Angew. Chem. Int. Ed. 49, 9233-9236 (2010).

32. Wang, H., Yan, Y., Li, B., Bi, L. \& Wu, L. Hierarchical self-assembly of surfactantencapsulated and organically grafted polyoxometalate complexes. Chem. Eur. J. 17, 4273-4282 (2011).

33. Nisar, A. \& Wang, X. Surfactant-encapsulated polyoxometalate building blocks: Controlled assembly and their catalytic properties. Dalton Trans. 41, 9832-9845 (2012).

34. Lin, X., Wang, Y. \& Wu, L. Hexagonal mesostructure and its disassembly into nanofibers of a diblock molecule/polyoxometalate hybrid. Langmuir 25, 6081-6087 (2009)

35. Nisar, A., Lu, Y. \& Wang, X. Assembling polyoxometalate clusters into advanced nanoarchitectures. Chem. Mater. 22, 3511-3518 (2010).

36. Nisar, A., Lu, Y., Zhuang, J. \& Wang, X. Polyoxometalate nanocone nanoreactors: Magnetic manipulation and enhanced catalytic performance. Angew. Chem. Int. Ed. 50, 3187-3192 (2011)

37. Li, C. et al. Synthesis and characterization of biocompatible thermo-responsive gelators based on aba triblock copolymers. Biomacromolecules 6, 994-999 (2005).

38. McCann, J. T., Chen, J. I. L., Li, D., Ye, Z.-G. \& Xia, Y. Electrospinning of polycrystalline barium titanate nanofibers with controllable morphology and alignment. Chem. Phys. Lett. 424, 162-166 (2006).

39. Andreas, G. \& Joachim, H. W. Electrospinning: a fascinating method for the preparation of ultrathin fibers. Angew. Chem. Int. Ed. 46, 5670-5703 (2007).

40. Lyon, D. K. et al. Highly oxidation resistant inorganic-porphyrin analog polyoxometalate oxidation catalysts. 1 . The synthesis and characterization of aqueous-soluble potassium salts of .Alpha.2- $\mathrm{P}_{2} \mathrm{~W}_{17} \mathrm{O}_{61}\left(\mathrm{M}^{\mathrm{n}} \cdot \text {. cntdot. } \mathrm{OH}_{2}\right)^{(\mathrm{n}-10)}$ and organic solvent soluble tetra-n-butylammonium salts of .Alpha.2$\mathrm{P}_{2} \mathrm{~W}_{17} \mathrm{O}_{61}\left(\mathrm{M}^{\mathrm{n}+} \text {.cntdot. } \mathrm{Br}\right)^{(\mathrm{n}-11)}\left(\mathrm{M}=\mathrm{Mn}^{3+}, \mathrm{Fe}^{3+}, \mathrm{Co}^{2+}, \mathrm{Ni}^{2+}, \mathrm{Cu}^{2+}\right)$. J. Am. Chem. Soc. 113, 7209-7221 (1991)

41. Finke, R. G., Rapko, B., Saxton, R. J. \& Domaille, P. J. Trisubstituted heteropolytungstates as soluble metal oxide analogs. Iii. Synthesis, characterization, phosphorus-31, silicon-29, vanadium-51, and 1- and 2-D tungsten-183 NMR, deprotonation, and proton mobility studies of organic 
solvent solute forms of $\mathrm{H}_{\mathrm{x}} \mathrm{SiW}_{9} \mathrm{~V}_{3} \mathrm{O}_{40}{ }^{\mathrm{x}-7}$ and $\mathrm{H}_{\mathrm{x}} \mathrm{P}_{2} \mathrm{~W}_{15} \mathrm{~V}_{3} \mathrm{O}_{62}{ }^{\mathrm{x}-9}$. J. Am. Chem. Soc. 108, 2947-2960 (1986).

42. Graham, C. R. \& Finke, R. G. The classic wells-dawson polyoxometalate, $\mathrm{K}_{6}\left[\alpha-\mathrm{P}_{2} \mathrm{~W}_{18} \mathrm{O}_{62}\right] \cdot 14 \mathrm{H}_{2} \mathrm{O}$. Answering an 88 year-old question: What is its preferred, optimum synthesis? Inorg. Chem. 47, 3679-3686 (2008).

43. $\mathrm{Wu}, \mathrm{H}$. Contribution to the chemistry of phosphomolybdic acids, phosphotungstic acids, and allied substances. J. Biol. Chem. 43, 189-220 (1920).

\section{Acknowledgements}

This work was supported by NSFC (91127040, 20971078, 21221062), and the State Key Project of Fundamental Research for Nanoscience and Nanotechnology (2011CB932402).

\section{Author contributions}

P.L.H. carried out the experimental work, analyzed the data and wrote the paper. B.X., H.L.L., S.H., and F.S. helped with the design and experiments. X.W. conceived the whole experimental project, analyzed the data and wrote the paper.

\section{Additional information}

Supplementary information accompanies this paper at http://www.nature.com/ scientificreports

Competing financial interests: The authors declare no competing financial interests. License: This work is licensed under a Creative Commons

Attribution-NonCommercial-NoDerivs 3.0 Unported License. To view a copy of this license, visit http://creativecommons.org/licenses/by-nc-nd/3.0/

How to cite this article: He, P. et al. Polyoxometalate-based Supramolecular Gel. Sci. Rep. 3, 1833; DOI:10.1038/srep01833 (2013). 